\title{
Increased Heart Rate Variability Response Among Infants with Reported Rhinorrhea and Watery Eyes: A Pilot Study
}

\author{
Laura A Conrad (1D \\ Natalie Buchinsky ${ }^{2}$ \\ Luis $M$ Acosta $^{2}$ \\ J David Nugent ${ }^{3}$ \\ Khalil W Savary iD ${ }^{4}$ \\ Rachel L Miller 5 \\ Nurdant Emanet ${ }^{6}$ \\ Julie Herbstman ${ }^{2}$ \\ Beatrice Beebe ${ }^{6}$ \\ Michael M Myers ${ }^{3}$ \\ William P Fifer ${ }^{3}$ \\ Matthew S Perzanowski iD ${ }^{2}$ \\ 'Division of Respiratory and Sleep \\ Medicine, Albert Einstein College of \\ Medicine, New York, NY, USA; \\ ${ }^{2}$ Department of Environmental Health \\ Sciences, Mailman School of Public \\ Health, Columbia University, New York, \\ NY, USA; ${ }^{3}$ Division of Developmental \\ Neuroscience, Department of Psychiatry, \\ Columbia University College of \\ Physicians and Surgeons, New York, NY, \\ USA; ${ }^{4}$ Department of Pediatrics, Rutgers \\ University, Newark, NJ, USA; ${ }^{5}$ Division of \\ Clinical Immunology, Department of \\ Medicine, Icahn School of Medicine at \\ Mount Sinai, New York, NY, USA; \\ ${ }^{6}$ Department of Psychiatry, Columbia \\ University College of Physicians and \\ Surgeons, New York, NY, USA
}

Correspondence: Matthew S Perzanowski Department of Environmental Health Sciences, Mailman School of Public Health, Columbia University, 722 West I68th St, I Ith Floor, New York, NY, 10032, USA

Tel +1 2/2-305-3465

$\mathrm{Fax}+1$ 212-305-4012

Email mp2217@cumc.columbia.edu
Introduction: Previously, we found that reported infant rhinorrhea and watery eyes without a cold (RWWC) predicted school age exercise-induced wheeze, emergency department visits, and hospitalizations. These findings were independent of allergic sensitization, and we theorized that increased parasympathetic tone underlay the association. We also reported that increased heart-rate variability (HRV) in infants predicted wheeze in 2-3 year-olds. In a convenience sample of children participating in a birth cohort study, we tested the hypothesis that infants with RWWC would have elevated HRV, indicating increased parasympathetic tone.

Methods: RWWC symptoms since birth were queried for 3-month-old children. At 4-months, HRV was assessed (root mean square of successive differences [RMSSD]) during a standardized infant-mother still-face paradigm, which included 2 minutes of mother/child play immediately followed by 2 minutes of the mother maintaining a still-face.

Results: Among participants ( $\mathrm{n}=38)$, RWWC was common for girls $(32 \%)$ and boys $(21 \%)$. The children with the greatest decrease in RMSSD between play and still-face challenge (lowest tertile) had a higher prevalence of RWWC as compared with children in the higher tertiles ( $50 \%$ vs $16 \%, \mathrm{P}=0.045)$. In a logistic regression model controlling for sex, age and time between HRV and RWWC assessment, children with greater decrease in HRV between play and still-face (lowest tertile) had greater odds of having RWWC (odds ratio=6.0, $\mathrm{P}=0.029$ ).

Conclusion: In this relatively small study, we demonstrated greater decreases in HRV in response to a stressor among children with reported RWWC, suggesting that these children might have increased parasympathetic tone and/or overall greater vagal reactivity.

Keywords: watery eyes, still-face challenge, rhinitis, exercise-induced asthma, autonomic nervous system, heart rate variability

\section{Plain Language Summary}

Previously, we observed an association between exercise-induced wheeze asthma phenotype, independent of allergic sensitization, and urgent healthcare utilization for asthma among children in urban neighborhoods. This led to the further evaluation of mechanisms underlying this connection, including the autonomic nervous system's role in exercise-induced wheeze. Rhinitis and watery eyes are well-known risk factors for development of asthma, which is commonly thought to be through an allergic pathway. However, the autonomic nervous system has been shown to be a cause of rhinitis and watery eyes due to increased response of the parasympathetic nervous system. We previously found that infant rhinorrhea and watery eyes reported without a cold (RWWC), predicted school age exercise-induced 
wheeze, emergency department visits, and hospitalizations independent of allergy. We also found that increased heart rate variability in infants predicted wheeze in toddlers. We therefore hypothesized that infants with rhinorrhea and watery eyes reported without a cold would have elevated heart rate variability.

In this study, we evaluated 38 infants. Parents reported on rhinorrhea and watery eyes without a cold. The infant's heart rate variability was measured during a structured protocol that assessed infant stress response and autonomic nervous system function. We found greater decreases in heart rate variability among those infants with a report of RWWC as compared to infants without RWWC suggesting a possible increase in parasympathetic tone. This study adds to a growing body of literature to suggest a connection between infant autonomic nervous system dysregulation and school age wheeze independent of the allergic asthma pathway.

\section{Introduction}

Rhinitis and watery eyes are well-known risk factors for asthma development. ${ }^{1,2}$ Although this link is thought to occur primarily through a common allergic pathway, studies have shown that non-allergic pathways related to parasympathetic nervous system (PNS) signaling can cause rhinorrhea and watery eyes through airway cooling (vasomotor rhinitis) and increased PNS response (rhinorrhea and watery eyes). ${ }^{3,4}$

We found that, among New York City (NYC) children, the occurrence of rhinorrhea and/or watery eyes without a cold (RWWC) in infancy predicted school-age exerciseinduced wheeze (EIW), emergency department (ED) visits for asthma, and hospitalizations for difficulty breathing. ${ }^{5}$ These findings were independent of allergic sensitization. In addition, among the same children we observed that prenatal exposure to pesticides and maternal stress, both of which can increase PNS signaling in infants, ${ }^{6,7}$ predicted infant RWWC. ${ }^{8,9}$ Furthermore, we observed that an indicator of increased PNS activity, increased heart rate variability (HRV), measured in infancy predicted subsequent wheeze at age 2-3 years among children in a birth cohort in South Dakota. ${ }^{10}$ Most recently, we have observed that children wheezing at age 2-3 years had higher HRV 1-3 years later, suggesting a persistence of increased PNS activity. $^{11}$

Based on these findings, we hypothesized that infants with RWWC would have elevated HRV, indicating increased parasympathetic tone. We selected the root mean square of successive differences (RMSSD) and change in RMSSD as the primary outcomes because
RMSSD is a time domain measure of HRV commonly used to estimate vagally mediated changes in HRV and thus reflects parasympathetic modulation. ${ }^{12}$ We tested this hypothesis among children participating in a prospective birth cohort study in NYC who underwent a standardized physiological challenge, the infant-mother still-face challenge.

\section{Materials and Methods}

A convenience sample of children were enrolled from the Fair Start Birth Cohort Study, an Environmental Influences on Child Health Outcomes study in NYC. Columbia University's Institutional Review Board approved this study and it was conducted in accordance with the Declaration of Helsinki. Consenting mothers were informed about the purpose of the study.

At approximately 3 months of age, reports by the parent or guardian of RWWC symptoms were ascertained for the children since birth. Within 2 months of completing this questionnaire, we examined the HRV response to a standardized physiological challenge, the infant-mother still-face paradigm. ${ }^{13}$ HRV was measured during the standardized face-to-face still-face paradigm. Research workers attached a Zephyr BioPatch (Medtronic Inc, Parsippany, NJ) to the child's chest using the Zephyr Biopatch Side Strap before the challenge. The still-face challenge included successive episodes of 1) 2 minutes of mother/infant play, 2) 2 minutes during which the mother maintained a still-face, refraining from talking with, touching or smiling at the infant, and 3) a 2-minute reunion period where the mother resumes normal interactions with the child. ${ }^{13}$ The root mean square of successive differences (RMSSD) in inter-beat-intervals was calculated for each of the challenge episodes using methods described previously. ${ }^{14}$ RMSSD and changes in RMSSD were selected as the primary measures of HRV. We tested for differences in RMSSD among children with and without RWWC during each period and the change between play and the still-face period.

\section{Results}

Among participants $(n=38)$, the mean age when the RWWC questionnaire was administered was 116 days (Table 1). The mean age at the still-face challenge was 135 days. The average time between administering the questionnaire and the still-face challenge was 19 days. Report of RWWC was common for girls (32\%) and boys $(21 \%)$. 
Table I Characteristics and HRV Outcomes Among Children Without and With RWWC

\begin{tabular}{|c|c|c|c|}
\hline & \multicolumn{2}{|c|}{$\begin{array}{l}\text { Report of Rhinorrhea and/or Watery Eyes } \\
\text { without Cold }\end{array}$} & \multirow[t]{2}{*}{$P$ value } \\
\hline & No $(n=28)$ & Yes $(n=10)$ & \\
\hline Female sex, $\mathrm{n}[\%]$ & $13[46]$ & $6[60]$ & 0.46 \\
\hline Mean age at RWWC assessment (days) [minimum - maximum] & $118[92-142]$ & $112[86-144]$ & 0.41 \\
\hline Mean age at HRV assessment (days) [minimum - maximum] & $135[125-157]$ & $136[126-144]$ & 0.59 \\
\hline Time between assessment of RWWC and HRV (days) [minimum - maximum] & $17[0-51]$ & $24[0-55]$ & 0.31 \\
\hline Median RMSSD during play episode (ms) & 0.012 & 0.014 & 0.40 \\
\hline Median RMSSD during still-face episode (ms) & 0.010 & 0.011 & 0.81 \\
\hline Median change in RMSSD between play and still face episodes (ms) & 0.0 & -0.0036 & 0.047 \\
\hline
\end{tabular}

The median RMSSD during the baseline play episode and the still-face episode were not statistically significantly different among children with and without RWWC (Table 1). The median change in RMSSD was statistically significantly lower (ie, greater decrease) among the children with as compared to children without RWWC $(\mathrm{P}=0.047)$.

The prevalence RWWC was not statistically significantly different among children with the lowest, middle or highest tertile of RMSSD during the mother/infant play episode $(17 \%, 31 \%, 31 \%$, respectively, $\mathrm{P}=0.66)$, nor during the 2 minutes episode of the mother maintaining a stillface $(17 \%, 43 \%, 17 \%, \mathrm{P}=0.21)$. However, the children with the greatest decrease in RMSSD between play and still-face challenge had a higher prevalence of RWWC (Figure 1). In a logistic regression model controlling for sex, HRV assessment age and time between HRV and RWWC assessment, children in the tertile with the greatest decrease in HRV between play and still-face had greater odds of having RWWC as compared with the other two tertiles of children $[\mathrm{OR}=6.0(95 \% \mathrm{CI}=1.2-30), \mathrm{P}=0.029]$.

\section{Discussion}

We observed that infants with increased HRV response were more likely to have had a report of rhinorrhea and/ or watery eyes symptoms in the absence of a cold. These findings suggest that PNS signaling may underlie infant RWWC for some children. Based on our previous findings and those from this pilot study, it appears as though this altered autonomic nervous system (ANS) activity in infancy manifests as RWWC and predicts the development of exercise-induced wheeze (EIW) at school age (Figure 2) as we describe in greater detail below.

Although regulation of the ANS is key to asthma pathophysiology (eg, vagal control of bronchial smooth muscle tone, blockage of bronchial hyperreactivity with anti- cholinergic agents), the importance of ANS dysregulation to asthma pathogenesis is underappreciated. ${ }^{7,15,16}$ For example, "nerves" are not mentioned in a recently released 322-page Guidelines for Diagnosing and Management of Asthma. ${ }^{16,17}$ EIW, which is both a specific asthma phenotype and a symptom indicating suboptimal control of asthma, may be a manifestation of ANS dysfunction related to increased activity in the parasympathetic arm of the ANS in response to dehydration and osmolar changes. ${ }^{18,19}$ Our interest in studying an ANS pathway leading to EIW came from observations of associations between EIW and urgent medical visits for asthma among NYC children that were independent of established markers of asthma severity, including lung function and frequent asthma symptoms,

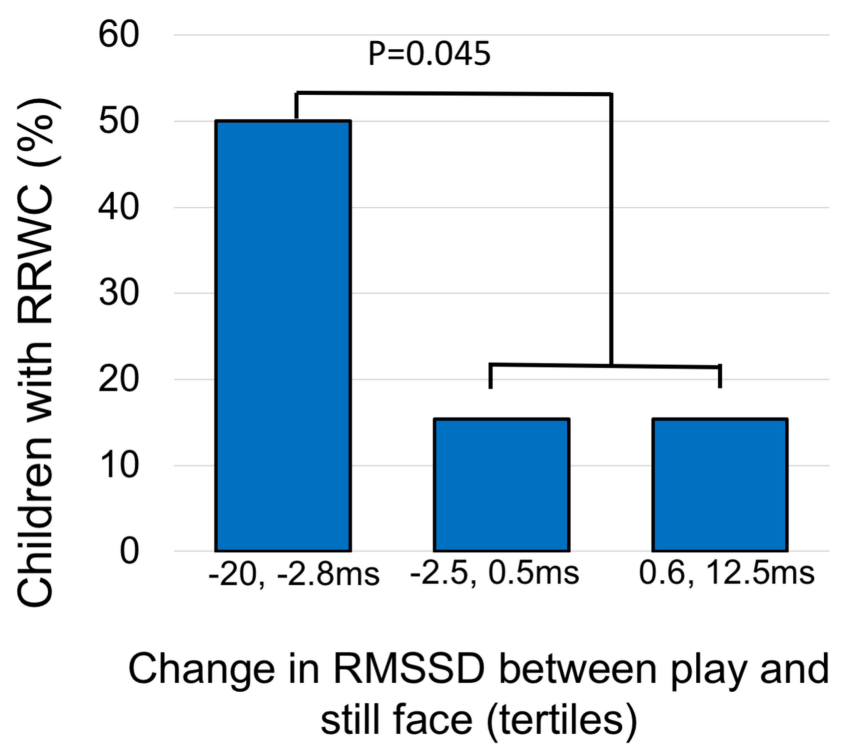

Figure I Prevalence of rhinorrhea/watery eyes without colds (RWWC) at age 3 months by tertile of change in heart rate variability (root mean square of the successive differences: RMSSD) measured between play and still-face challenge $(n=38)$. Children with the greatest decrease in HRV were the most likely to have had a report of RWWC. 


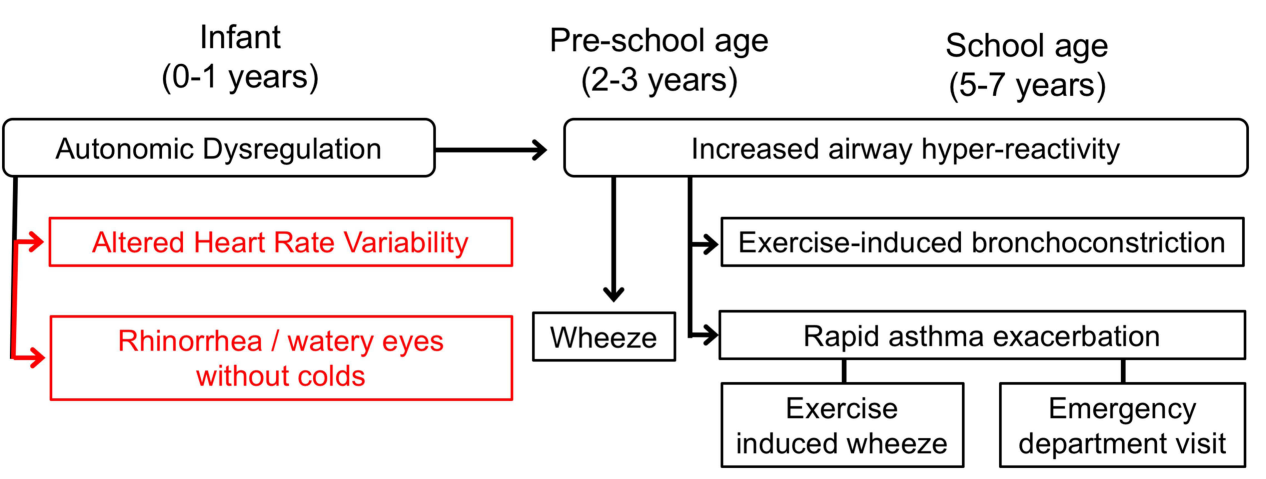

Figure 2 Hypothesized connection between autonomic dysregulation in infancy and airway hyperreactivity at school age. Components tested in this analysis are shown in red.

and not related to allergic sensitization. ${ }^{5,20}$ Collectively, these findings suggested that an EIW phenotype, independent of allergy, could account for a significant proportion of the ED visits and hospitalizations for asthma that afflict children living in lower-income neighborhoods. ${ }^{20}$

Rhinitis and watery eyes are well-known risk factors for subsequent asthma development. ${ }^{2}$ Although the link between rhinitis and watery eyes and subsequent asthma is thought to occur primarily through a common allergic pathway, studies have shown that non-allergic pathways related to parasympathetic nervous system (PNS) signaling can cause rhinitis and watery eyes through airway cooling (vasomotor rhinitis) and increased PNS response (rhinorrhea and watery eyes). ${ }^{3,4}$ Therefore, rhinitis, watery eyes, and EIW may share physiological responses independent of an allergic immune response. Among children participating in the Columbia Center for Children's Environmental Health prospective birth cohort of children living in lower-income NYC communities, we found that the occurrence of RWWC in infancy predicted school-age EIW (relative risk $[R R]=2.8, P<0.001)$, $E D$ visits $(R R=1.8$, $\mathrm{P}=0.001$ ), and hospitalizations ( $\mathrm{RR}=9.8, \mathrm{P}=0.002)$, independent of allergic sensitization. ${ }^{5}$ In addition, infant RWWC was predicted by prenatal exposure to pesticides $(\mathrm{P}<0.01)$ and maternal stress $(\mathrm{P}<0.01)$, both of which can alter PNS signaling in infants. ${ }^{7,91}$ Furthermore, we recently reported that increased high-frequency heart rate variability (HF-HRV), an indicator of increased PNS activity, measured in infancy, predicted wheeze at age 2-3 years in children. ${ }^{22}$ Our current study provides additional evidence to suggest that infants with altered PNS signaling may manifest this through increased HRV and RWWC, which in turn could potentially serve as a means to identify and survey young children at greater risk of asthma symptoms (Figure 2).
We are unaware of other studies investigating the ANS and infant rhinorrhea. A relatively small study of older children (mean age 12 years) compared children with allergic rhinitis to healthy controls and found that children with allergic rhinitis had statistically significantly higher RMSSD and HF-HRV. ${ }^{23}$ A second study of children aged 7-12 years also found that children with allergic rhinitis had higher heart rate variability measures associated with parasympathetic control. ${ }^{24}$ Both studies found inverse correlations between rhinitis symptom frequency and HRV. Similarly, in a study among adults, individuals with allergic rhinitis had statistically significantly higher HF-HRV than healthy controls, and total nasal symptoms were inversely correlated with HF-HRV. ${ }^{25}$ It is important to point out that these latter studies did not examine children or adults with non-allergic rhinitis. It has been hypothesized that some phenotypes of non-allergic rhinitis have underlying mechanisms with increased PNS responsiveness or an imbalance of the PNS and sympathetic nervous system. ${ }^{26} \mathrm{~A}$ study in adults examined vasomotor rhinitis, a type of non-allergic rhinitis, and found increased indicators of the PNS responsiveness. ${ }^{27}$ Because inhalant allergen sensitization develops later in childhood, it is unlikely that the children in our study have allergy to inhalant allergens. Also, our prior findings showing that infant RWWC predicted school age EIW was observed independent of allergic sensitization status. ${ }^{5}$ Therefore, there is some compelling evidence connecting rhinorrhea to increased PNS responsiveness, but more work needs to be done to understand the relevance in infancy and among non-allergic rhinitis.

A limitation of this study was that RWWC was ascertained via questionnaire. Diagnostic testing or physician assessment to rule out infectious etiologies of RWWC were not performed. Thus, children with rhinorrhea and a cold 
could have been misclassified as having RWWC. However, in a previous study of children in NYC, we reported that in the first year of life, $32 \%$ of the children had a report of runny nose without a cold, while virtually all of the children (98\%) had a report of runny nose with a cold for the same time period. ${ }^{5}$ As we describe in a previous paragraph, infant RWWC in that cohort was predicted by exposures that can modulate an infant's ANS (eg, maternal stress and pesticide exposure) and also predicted subsequent exercise induced asthma, which may have an underlying PNS mechanism. These prior findings and the findings from this relatively small pilot study support RWWC as a symptom of interest in future research studies even though it is not a clinically relevant outcome on its own. Future studies should test for current infection during episodes of RWWC to validate the lack of current infection.

There are other limitations of the study. The timing of questionnaire data on RWWC and HRV data collection varied among patients within a 2-month window. It is possible that an episode of RWWC may influence HRV data. The population studied comes from communities with high risk of developing asthma, EIW and emergency department visits for asthma. As such, the findings from this study may not be generalizable to other populations. Importantly, this is a pilot study with a relatively small sample size. The findings need to be confirmed in future studies with a larger sample size.

\section{Conclusions}

In this pilot study, we demonstrated greater decreases in $\mathrm{HRV}$ in response to a stressor among children with reported RWWC, suggesting that these children might have increased parasympathetic tone and/or overall greater vagal reactivity.

\section{Abbreviations}

ANS, autonomic nervous system; CCCEH, Columbia Center for Children's Environmental Health; ED, emergency department; ETS, environmental tobacco smoke; HF-HRV, high frequency variation in heart rate; HRV, heart rate variability; $\mathrm{PR}$, prevalence ratio; $\mathrm{RR}$, relative risk; RWWC, rhinorrhea and watery eyes without a cold.

\section{Data Sharing Statement}

A dataset specific to the results presented in this manuscript will be made available upon request after stripping identifiers prior to release. However, we believe that there remains the possibility of deductive disclosure of subjects with unusual characteristics. Thus, we will make the data and associated documentation available to users only under a data-sharing agreement that provides for (1) a commitment to using the data only for research purposes and not to identify any individual participant; (2) a commitment to securing the data using appropriate computer technology; and (3) a commitment to destroying or returning the data after analyses are completed.

\section{Ethics Approval and Informed Consent}

This study was approved by Institutional Review Boards at both Columbia University's Medical Center and the New York State Psychiatric Institute. Mothers consented to participate and we were able to leave the study at any time.

\section{Consent for Publication}

All co-authors have reviewed this manuscript and consent to its publication.

\section{Acknowledgments}

The authors would like to thank the participating mothers and children. This work would not have been possible without the hard work and dedication of the research workers and field technicians. Data from this paper was presented at the 2019 American Academy of Asthma Allergy and Immunology as a poster presentation with interim findings. The poster's abstract was published with other meeting abstracts in the Journal of Allergy and Clinical Immunology 2019;143;2:Supplement AB81. DOI: https://www.jacionline.org/article/S0091-6749(18) 31993-6/fulltext

\section{Author Contributions}

All authors made substantial contributions to conception and design, acquisition of data, or analysis and interpretation of data; took part in drafting the article or revising it critically for important intellectual content; agreed to submit to the current journal; gave final approval of the version to be published; and agree to be accountable for all aspects of the work.

\section{Funding}

This work was supported by the National Institute of Health [grant numbers 5R01ES027424-02, 5UH3OD023290-04 and P30 ES009089] and the Mailman School of Public Health [pilot grant]. 


\section{Disclosure}

The authors have no competing interests related to this manuscript.

\section{References}

1. Rzehak P, Schoefer Y, Wichmann HE, Heinrich J. A prospective study on the association between hay fever among children and incidence of asthma in East Germany. Eur J Epidemiol. 2008;23 (1):17-22. doi:10.1007/s10654-007-9205-3

2. Shaaban R, Zureik M, Soussan D, et al. Rhinitis and onset of asthma: a longitudinal population-based study. Lancet. 2008;372 (9643):1049-1057. doi:10.1016/S0140-6736(08)61446-4

3. van der Velden VH, Hulsmann AR. Autonomic innervation of human airways: structure, function, and pathophysiology in asthma. Neuroimmunomodulation. 1999;6(3):145-159. doi:10.1159/ 000026376

4. Kardon R. Anatomy and Physiology of the Autonomic Nervous System. 6th ed. Philadelphia, PA, USA: Lippincott Williams \& Wilkins; 2005.

5. Savary KW, Miller RL, Arteaga-Solis E, et al. Infant rhinitis and watery eyes predict school-age exercise-induced wheeze, emergency department visits and respiratory-related hospitalizations. Ann Allergy Asthma Immunol. 2018;120(3):278-284 e272. doi:10.1016/j. anai.2017.11.024

6. Young JG, Eskenazi B, Gladstone EA, et al. Association between in utero organophosphate pesticide exposure and abnormal reflexes in neonates. Neurotoxicology. 2005;26(2):199-209. doi:10.1016/j. neuro.2004.10.004

7. Wright RJ. Stress-related programming of autonomic imbalance: role in allergy and asthma. Chem Immunol Allergy. 2012;98:32-47.

8. Conrad LA, Buchinsky N, Acosta LM, et al. Infant rhinorrhea and watery eyes in the absence of a cold associated with increased heart rate variability among girls. J Allergy Clin Immunol. 2020;145(2): AB13. doi:10.1016/j.jaci.2019.12.869

9. Perzanowski MS, Rauh VA, Conrad LA, et al. Report of prenatal exposure to pesticide predicts infant rhinitis and watery eyes without a cold. J Allergy Clin Immunol. 2019;143(2):AB81. doi:10.1016/j. jaci.2018.12.251

10. Dukes KA, Burd L, Elliott AJ, et al. The safe passage study: design, methods, recruitment, and follow-up approach. Paediatr Perinat Epidemiol. 2014;28(5):455-465. doi:10.1111/ppe.12136

11. Berger S, Nugent DJ, Acosta LM, et al. Pre-school age wheeze associated with increased heart rate variability measured $1-3$ years later among girls. AJRCCM. 2021;203:A3246.

12. Shaffer F, Ginsberg JP. An overview of heart rate variability metrics and norms. Front Public Health. 2017;5:258. doi:10.3389/ fpubh.2017.00258

13. Barbosa M, Beeghly M, Moreira J, Tronick E, Fuertes M. Robust stability and physiological correlates of infants' patterns of regulatory behavior in the still-face paradigm at 3 and 9 months. Dev Psychol. 2018;54(11):2032-2042. doi:10.1037/dev0000616
14. Myers MM, Elliott AJ, Odendaal HJ, et al. Cardiorespiratory physiology in the safe passage study: protocol, methods and normative values in unexposed infants. Acta Paediatr. 2017;106(8):1260-1272. doi:10.1111/apa.13873

15. Goyal M, Jaseja H, Verma N. Increased parasympathetic tone as the underlying cause of asthma: a hypothesis. Med Hypotheses. 2010;74 (4):661-664. doi:10.1016/j.mehy.2009.11.011

16. Canning BJ, Woo A, Mazzone SB. Neuronal modulation of airway and vascular tone and their influence on nonspecific airways responsiveness in asthma. J Allergy. 2012;2012:108149.

17. National Heart, Lung, and Blood Institute. Asthma Management Guidelines: Focused Updates 2020. Available from:https://www. nhlbi.nih.gov/health-topics/asthma-management-guidelines-2020updates. Accessed October 28, 2021.

18. Papadopoulos NG, Arakawa $\mathrm{H}$, Carlsen $\mathrm{KH}$, et al. International consensus on (ICON) pediatric asthma. Allergy. 2012;67 (8):976-997. doi:10.1111/j.1398-9995.2012.02865.x

19. Carlsen KH, Anderson SD, Bjermer L, et al. Exercise-induced asthma, respiratory and allergic disorders in elite athletes: epidemiology, mechanisms and diagnosis: part I of the report from the joint task force of the European Respiratory Society (ERS) and the European Academy of Allergy and Clinical Immunology (EAACI) in cooperation with GA2LEN. Allergy. 2008;63(4):387-403.

20. Mainardi TR, Mellins RB, Miller RL, et al. Exercise-induced wheeze, urgent medical visits, and neighborhood asthma prevalence. Pediatrics. 2013;131(1):e127-135. doi:10.1542/peds.2012-1072

21. Conrad LA, Rauh VA, Hoepner LA, et al. Report of prenatal maternal demoralization and material hardship and infant rhinorrhea and watery eyes. Ann Allergy Asthma Immunol. 2020;125(4):399-404 e392. doi:10.1016/j.anai.2020.07.016

22. Perzanowski MS, Savary KW, Arteaga-Solis E, et al. Associations between parasympathetic activity in the month after birth and wheeze at age 2-3 years. Am J Respir Crit Care Med. 2018;198(4):532-534. doi:10.1164/rccm.201712-2591LE

23. Tascilar E, Yokusoglu M, Dundaroz R, et al. Cardiac autonomic imbalance in children with allergic rhinitis. Tohoku J Exp Med. 2009;219(3):187-191. doi:10.1620/tjem.219.187

24. Emin O, Esra G, Ufuk E, Demiri A, Ayhan S, Rusen DM. Autonomic dysfunction and clinical severity of disease in children with allergic rhinitis. Int J Pediatr Otorhinolaryngol. 2012;76(8):1196-1200. doi:10.1016/j.ijporl.2012.05.005

25. Kim MH, Choi EJ, Jang BH, Kim KS, Ko SG, Choi I. Autonomic function in adults with allergic rhinitis and its association with disease severity and duration. Ann Allergy Asthma Immunol. 2017;118 (2):174-178. doi:10.1016/j.anai.2016.11.012

26. Sarin S, Undem B, Sanico A, Togias A. The role of the nervous system in rhinitis. J Allergy Clin Immunol. 2006;118(5):999-1016. doi:10.1016/j.jaci.2006.09.013

27. Jaradeh SS, Smith TL, Torrico L, et al. Autonomic nervous system evaluation of patients with vasomotor rhinitis. Laryngoscope. 2000;110(11):1828-1831. doi:10.1097/00005537-200011000-00012

\section{Publish your work in this journal}

The Journal of Asthma and Allergy is an international, peer-reviewed open-access journal publishing original research, reports, editorials and commentaries on the following topics: Asthma; Pulmonary physiology; Asthma related clinical health; Clinical immunology and the immunological basis of disease; Pharmacological interventions and new therapies. The manuscript management system is completely online and includes a very quick and fair peer-review system, which is all easy to use. Visit http://www.dovepress.com/testimonials.php to read real quotes from published authors. 